TAMKANG JOURNAL OF MATHEMATICS

Volume 33, Number 1, Spring 2002

\title{
MATRIX SUMMABILITY OF THE CONJUGATE SERIES OF DERIVED FOURIER SERIES
}

\author{
SHYAM LAL AND PURNIMA YADAV
}

\begin{abstract}
In this paper, a new theorem on matrix summability of the conjugate series of a derived Fourier series is proved, which improves and generalizes all the previous known results in this line of work.
\end{abstract}

\section{Introduction}

There are several known results on Nörlund summability of Fourier series, conjugate series and derived series of Fourier series. In 1963, for the first time Tripathi [5] established a theorem for Harmonic summablity of conjugate series of derived Fourier series. Later on in 1972, Tripathi and Prasad [6] generalized the result of Tripathi for $\left(N, p_{n}\right)$ summability which is weaker than Harmonic summability. Working in the same direction Tripathi \& Singh [8], Tripathi \& Prasad [7] and Sulaimann [3] studied $\left(N, p_{n}\right)$ summability of conjugate series of derived Fourier series series and conjugate series of Fourier series by matrix means. But until now no work seems to have been done in the direction of a study of conjugate series of derived Fourier series by matrix summability. It is important to note that Cesáro, Harmonic, Riesz, Nörlund and generalized Nörlund summabilities are particular cases of the matrix summability. The purpose of this paper is to establish a more general result than those of Tripathi [5], Tripathi \& Prasad [6], Tripathi \& Singh [8] and Tripathi \& Prasad [7].

\section{Definitions and Notations}

Let $\sum u_{n}$ be a given infinite series with sequence of partial $\operatorname{sum}\left\{S_{n}\right\}$. Let $T=\left(a_{n, k}\right)$ be an infinite triangular matrix of real constants. The sequence-to-sequence transformation

$$
t_{n}=\sum_{k=0}^{n} a_{n, k} S_{k}=\sum_{k=0}^{n} a_{n, n-k} S_{n-k}
$$

Received March 02, 2001; revised June 01, 2001.

2000 Mathematics Subject Classification. Primary 42B05, $42 \mathrm{~B} 08$.

Key words and phrases. Matrix summability, derived Fourier series, conjugate derived Fourier series, $\left(N, p_{n}\right),(C, \alpha),\left(N, \frac{1}{n+1}\right)$ summabilities, $n^{\text {th }}$ partial sum, sequence \& monotonic decreasing function. 
defines the sequence $\left\{t_{n}\right\}$ of matrix means of the sequence $\left\{S_{n}\right\}$, generated by the sequence of coefficients $\left(a_{n, k}\right)$. The series $\sum u_{n}$ is said to be summable to the sum $S$ by matrix method if $\lim _{n \rightarrow \infty} t_{n}$ exists and is equal to $S$ (see Zygmund [9]).

We can write

$$
t_{n} \rightarrow S(T), \text { as } n \rightarrow \infty
$$

The necessary and sufficient conditions for $T$-transform to be regular $\left(i . e . \lim _{n \rightarrow \infty} S_{n} \rightarrow\right.$ $S \Rightarrow \lim _{n \rightarrow \infty} t_{n} \rightarrow S$ ) are the well-known Silverman-Toeplitz conditions. If $a_{n, k}=0$, for every $k>n$, then the matrix is called triangular (Toeplitz [4]).

Let $f(t)$ be a periodic function with period $2 \pi$, integrable in the sense of Lebesgue over $(-\pi, \pi)$. The Fourier series and conjugate series of $f(t)$ are given by

$$
f(t) \sim \frac{1}{2} a_{0}+\sum_{n=1}^{\infty}\left(a_{n} \cos n t+b_{n} \sin n t\right)
$$

and

$$
\sum_{n=1}^{\infty}\left(b_{n} \cos n t-a_{n} \sin n t\right) .
$$

Then the conjugate series of derived Fourier series is

$$
-\sum_{n=1}^{\infty} n\left(a_{n} \cos n t+b_{n} \sin n t\right)
$$

We shall use following notations:

$$
\begin{aligned}
h(t) & =[f(x+t)+f(x-t)-2 f(x)] \\
H(x) & =-\frac{1}{4 \pi} \int_{0}^{\pi} h(t) \operatorname{cosec}^{2} \frac{t}{2} d t \\
M_{n}(t) & =\frac{1}{2 \pi} \sum_{k=0}^{n} a_{n, n-k} \frac{\cos \left(n-k+\frac{1}{2}\right) t}{\sin \frac{t}{2}} ; \\
\tau & =\text { Integral part of } \frac{1}{t}=\left[\frac{1}{t}\right] .
\end{aligned}
$$

Particular Cases: Seven important particular cases of matrix means are

(i) $(C, 1)$ means, when $a_{n, k}=\frac{1}{n+1}$.

(ii) Harmonic means, when $a_{n, k}=\frac{1}{(n-k+1) \log n}$.

(iii) $(C, \delta)$ means, when $a_{n, k}=\frac{\left(\begin{array}{c}n-k+\delta+1 \\ \delta-1\end{array}\right)}{\left(\begin{array}{c}n+\delta \\ \delta\end{array}\right) .}, 0 \leq \delta \leq 1$. 
(iv) $(H, p)$ means, when $a_{n, k}=\frac{1}{(\log )^{p-1}(n+1)} \prod_{q=0}^{p-1} \log ^{q}(k+1)$.

(v) Nörlund means, when $a_{n, k}=\frac{p_{n-k}}{P_{n}}$ where $\left\{p_{n}\right\}$ is a real non-negative monotonic non-increasing sequence such that $P_{n}=\sum_{k=0}^{n} p_{k} \rightarrow \infty$, as $n \rightarrow \infty$.

(vi) Riesz means $\left(\bar{N}, p_{n}\right)$, when $a_{n, k}=\frac{p_{k}}{P_{n}}$ where $\left\{p_{n}\right\}$ is a non-negative, non-decreasing sequence such that $P_{n}=\sum_{k=0}^{n} p_{k} \rightarrow \infty$, as $n \rightarrow \infty$.

(vii) Generalized Nörlund means $(N, p, q)$, when $a_{n, k}=\frac{p_{n-k} q_{k}}{R_{n}}$ where $\left\{p_{n}\right\}$ is a nonnegative monotonic non-increasing sequence and $\left\{q_{n}\right\}$ is a non-negative monotonic non-decreasing sequence such that $R_{n}=\sum_{k=0}^{n} p_{n-k} q_{k} \rightarrow \infty$, as $n \rightarrow \infty$.

\section{Known Theorem}

Tripathi [5] established the following theorem.

Theorem. The conjugate derived series of the Fourier series of a function $f(x)$ is summable by harmonic means to the sum

$$
-\frac{1}{4 \pi} \int_{0}^{\pi} h(t) \operatorname{cosec}^{2} \frac{t}{2} d t
$$

at every point $x$ at which this integral exists and

$$
H(t)=\int_{0}^{t}|d h(t)|=o\left(\frac{t}{\log 1 / t}\right) \text {, as } t \rightarrow+0 .
$$

\section{Main Theorem}

Many results are known for $(C, \alpha),\left(N, \frac{1}{n+1}\right),\left(N, p_{n}\right),\left(N, p_{n}^{\alpha}\right), \alpha>-1$ summabilities of conjugate series of derived Fourier series. The purpose of this paper is to establish a theorem on conjugate series of derived Fourier series by matrix summability so that various known results come out as particular cases of our main theorem. In fact, in this paper, we shall establish the following theorem.

Theorem Let $\left\{a_{n, k}\right\}_{k=0}^{\infty}$ be a real non-negative and non-decreasing sequence with respect to $k$ such that $T=\left(a_{n, k}\right)$ be an infinite triangular matrix with $a_{n, k} \geq 0, A_{n, \tau}=$ $\sum_{k=0}^{\tau} a_{n, n-k}, A_{n, n}=1$ for each $n \geq 0$. If

$$
\int_{0}^{t}|d h(u)|=o\left(\frac{t \xi(1 / t)}{\log 1 / t}\right), \text { as } t \rightarrow+0,
$$


then the conjugate series of the derived Fourier series (2.2) is sumable $(T)$ to the sum $-\frac{1}{4 \pi} \int_{0}^{\pi} h(t) \operatorname{cosec}^{2} / \frac{t}{2} d t$, provided $\xi(t)$ is a positive monotonic decreasing function of $t$ such that $\frac{t \xi(1 / t)}{\log 1 / t}$ increases monotonically as $t \rightarrow+0$.

\section{Lemma}

Our proof of the theorem needs some lemmas:

Lemma 5.1. Lal [1]

If $a_{n, k}$ is non-negative and non-decreasing with $k$ then for $0 \leq a \leq b \leq \infty, 0 \leq t \leq \pi$ and for any $n$, we have

$$
\left|\sum_{k=a}^{b} a_{n, n-k} e^{i(n-k) t}\right| \leq O\left(A_{n, \tau}\right) .
$$

Lemma 5.2. For $\frac{1}{n} \leq t \leq \delta<\pi$,

$$
M_{n}(t)=O\left(\frac{A_{n, \tau}}{t}\right), \tau \leq n
$$

Proof. Now by (2.3)

$$
\begin{aligned}
M_{n}(t) & \leq\left|\sum_{k=0}^{n} a_{n, n-k} \frac{\cos \left(n-k+\frac{1}{2}\right) t}{\sin \left(\frac{t}{2}\right)}\right| \\
& \leq \frac{1}{\sin (t / 2)}\left|\operatorname{Re} \sum_{k=0}^{n} a_{n, n-k} e^{i\left(n-k+\frac{1}{2}\right) t}\right| \\
& \leq \frac{\pi}{t}\left|R e \sum_{k=0}^{n} a_{n, n-k} e^{i(n-k) t} e^{i t / 2}\right| \quad \text { (by Jordan's lemma) } \\
& \leq \frac{\pi}{t}\left|\sum_{k=0}^{n} a_{n, n-k} e^{i(n-k) t}\right| \quad\left(\because\left|e^{i t / 2}\right| \leq 1\right) \\
& =\frac{\pi}{t} O\left(A_{n, \tau}\right) \quad \text { by lemma }(5.1) \\
& =O\left(\frac{A_{n, \tau}}{t}\right) .
\end{aligned}
$$

\section{Proof Of Main Theorem}

Let $S_{r}(x)$ denote the $r^{t h}$ partial sum of the series (2.2). Then

$$
S_{r}(x)=-\frac{1}{\pi} \int_{-\pi}^{\pi} f(u) \frac{d}{d x}\left(\sum_{v=0}^{r} v \sin v(u-x)\right) d u
$$




$$
\begin{aligned}
= & -\frac{1}{\pi} \int_{0}^{\pi} \frac{d}{d t}\left[\frac{\cos t / 2-\cos \left(r+\frac{1}{2}\right) t}{\sin t / 2}\right]\{f(x+t)-f(x-t)\} d t \\
= & -\frac{1}{\pi} \int_{0}^{\pi} \frac{\cos t / 2-\cos \left(r+\frac{1}{2}\right) t}{\sin t / 2} d h(t) \\
= & -\frac{1}{2 \pi}\left[\int_{0}^{1 / n}+\int_{1 / n}^{\pi}\right]\left\{\cot \frac{t}{2}(1-\cos r t)+\sin r t\right\} d h(t), r<n . \\
= & -\frac{1}{2 \pi} \int_{0}^{1 / n}\left[\cot \frac{t}{2}(1-\cos r t)+\sin r t\right] d h(t)-\frac{1}{2 \pi} \int_{1 / n}^{\pi} \cot \frac{t}{2} d h(t) \\
& +\frac{1}{2 \pi} \int_{1 / n}^{\pi}\left[\cot \frac{t}{2} \cos r t-\sin r t\right] d h(t) \\
= & I_{1}+I_{2}+\frac{1}{2 \pi} \int_{1 / n}^{\pi}\left[\cot \frac{t}{2} \cos r t-\sin r t\right] d h(t), \text { say. }
\end{aligned}
$$

We have

$$
\begin{aligned}
I_{1} & \leq \frac{1}{2 \pi} \int_{0}^{1 / n}\left|\left\{\cot \frac{t}{2}(1-\cos r t)+\sin r t\right\}\right||d h(t)| \\
& \leq \frac{1}{2 \pi} \int_{0}^{1 / n}\left|\cot \frac{t}{2} 2 \sin ^{2} \frac{r t}{2}\right||d h(t)|+\frac{1}{2 \pi} \int_{0}^{1 / n}|\sin r t||d h(t)| \\
& \leq \frac{r^{2}}{2 \pi} \int_{0}^{1 / n}\left|\cot \frac{t}{2} 2 \sin ^{2} \frac{t}{2}\right||d h(t)|+\frac{r}{2 \pi} \int_{0}^{1 / n}|\sin t||d h(t)| \quad(\because \sin n \theta \leq n \sin \theta) \\
& =\frac{r^{2}}{2 \pi} \int_{0}^{1 / n}|\sin t||d h(t)|+\frac{r}{2 \pi} \int_{0}^{1 / n}|\sin t||d h(t)| \\
& \leq \frac{r^{2}}{2 \pi} \int_{0}^{1 / n}|t||d h(t)|+\frac{r}{2 \pi} \int_{0}^{1 / n}|t||d h(t)| \quad(\because|\sin \theta| \leq|\theta|) \\
& \leq \frac{r}{2 \pi} \frac{r}{n} \int_{0}^{1 / n}|d h(t)|+\frac{1}{2 \pi} \cdot \frac{r}{n} \int_{0}^{1 / n}|d h(t)| \quad(\because r<n) \\
& \leq \frac{r}{2 \pi} \int_{0}^{1 / n}|d h(t)|+\frac{1}{2 \pi} \int_{0}^{1 / n}|d h(t)| \quad \text { by }(4.1) \\
& =\frac{r}{2 \pi} o\left(\frac{\xi(n)}{n \log n}\right)+\frac{1}{2 \pi} o\left(\frac{\xi(n)}{n \log n}\right) \quad \\
& =o(1)+o(1), \text { as } n \rightarrow \infty . \text { by hypothesis of the theorem } \\
& =o(1), n \rightarrow \infty .
\end{aligned}
$$

Next, consider $I_{2}$

$$
\begin{aligned}
I_{2} & =-\frac{1}{2 \pi} \int_{1 / n}^{\pi} \cot \frac{t}{2} d h(t) \\
& =-\frac{1}{2 \pi}\left[h(t) \cot \frac{t}{2}\right]_{\frac{1}{n}}^{\pi}-\frac{1}{4 \pi} \int_{1 / n}^{\pi} h(t) \operatorname{cosec}^{2} \frac{t}{2} d t
\end{aligned}
$$




$$
\begin{aligned}
& =o(1)+\frac{1}{2 \pi}\left[h\left(\frac{1}{n}\right) \frac{\cos \frac{1}{2 n}}{\frac{1}{2 n}} \cdot \frac{\frac{1}{2 n}}{\sin \frac{1}{2 n}}\right]-\frac{1}{4 \pi} \int_{1 / n}^{\pi} h(t) \operatorname{cosec}^{2} \frac{t}{2} d t \\
& =o(1)+\frac{1}{2 \pi} 2 n h\left(\frac{1}{n}\right)-\frac{1}{4 \pi} \int_{1 / n}^{\pi} h(t) \operatorname{cosec}^{2} \frac{t}{2} d t \\
& =o(1)+\frac{1}{\pi} n o\left(\frac{\xi(n)}{n \log n}\right)-\frac{1}{4 \pi} \int_{1 / n}^{\pi} h(t) \operatorname{cossec}^{2} \frac{t}{2} d t \quad \text { by }(4.1) \\
& =o(1)+o(1)-\frac{1}{4 \pi} \int_{0}^{\pi} h(t) \operatorname{cosec}^{2} \frac{t}{2} d t+\frac{1}{4 \pi} \int_{0}^{\frac{1}{n}} h(t) \operatorname{cosec}^{2} \frac{t}{2} d t
\end{aligned}
$$

Therefore, from (6.1), (6.2) and (6.3), we get

$$
\begin{aligned}
& S_{r}(x)-\left(-\frac{1}{4 \pi} \int_{0}^{\pi} h(t) \operatorname{cosec}^{2} \frac{t}{2} d t\right) \\
= & o(1)+\frac{1}{4 \pi} \int_{0}^{1 / n} h(t) \operatorname{cosec}^{2} \frac{t}{2} d t+\frac{1}{2 \pi} \int_{1 / n}^{\pi} \frac{\cos \left(r+\frac{1}{2}\right) t}{\sin t / 2} d h(t)
\end{aligned}
$$

Now

$$
\begin{aligned}
& \sum_{k=0}^{n} a_{n, n-k}\left[S_{n-k}(x)-\left(-\frac{1}{4 \pi} \int_{0}^{\pi} h(t) \operatorname{cosec}^{2} \frac{t}{2} d t\right)\right] \\
= & \sum_{k=0}^{n} a_{n, n-k}\left[o(1)+\frac{1}{4 \pi} \int_{0}^{1 / n} h(t) \operatorname{cosec}^{2} \frac{t}{2} d t+\frac{1}{2 \pi} \int_{1 / n}^{\pi} \frac{\cos \left(n-k+\frac{1}{2}\right) t}{\sin t / 2} d h(t)\right] .
\end{aligned}
$$

Next, $\quad t_{n}(x)-H(x)$

$$
=o(1)+\frac{1}{4 \pi} \int_{0}^{1 / n} h(t) \operatorname{cosec}^{2} \frac{t}{2} d+\int_{1 / n}^{\pi}\left[\frac{1}{2 \pi} \sum_{k=0}^{n} a_{n, n-k} \frac{\cos \left(n-k+\frac{1}{2}\right) t}{\sin t / 2}\right] d h(t)
$$

Since the conjugate series of the derived Fourier series is summable to $H(x)$, therefore

$$
-\frac{1}{4 \pi} \int_{0}^{1 / n} h(t) \operatorname{cosec}^{2} \frac{t}{2} d t=o(1), \quad \text { as } n \rightarrow \infty .
$$

Thus

$$
\begin{aligned}
& t_{n}(x)-H(x)=o(1)+o(1)+\int_{1 / n}^{\pi} M_{n}(t) d h(t) \\
= & o(1)+\left[\int_{1 / n}^{\delta}+\int_{\delta}^{\pi}\right] M_{n}(t) d h(t)
\end{aligned}
$$

where $\delta$ is a fixed positive number such that for $t \leq \delta$, condition (4.1) holds.

$$
=o(1)+I_{3}+I_{4} \text {, say. }
$$


Firstly let us consider $I_{3}$. Using lemma (5.2), we have

$$
\begin{aligned}
& I_{3} \leq \int_{1 / n}^{\delta}\left|M_{n}(t)\right||d h(t)| \\
& =O\left[\int_{1 / n}^{\delta} \frac{A_{n, \tau}}{t}|d h(t)|\right] \\
& =O\left[\frac{A_{n, \tau}}{t} o\left(\frac{t \xi(1 / t)}{\log 1 / t}\right)\right]_{1 / n}^{\delta}+O\left[\int_{1 / n}^{\delta} \frac{A_{n, \tau}}{t^{2}} o\left(\frac{t \xi(1 / t)}{\log 1 / t}\right) d t\right] \\
& +O\left[\int_{1 / n}^{\delta} \frac{1}{t} o\left(\frac{t \xi(1 / t)}{\log 1 / t}\right) d\left(A_{n, \tau}\right)\right] \\
& \text { (Integrating by parts and using condition (4.1)) } \\
& =o\left[A_{n, \tau} \frac{\xi(1 / t)}{\log 1 / t}\right]_{1 / n}^{\delta}+o\left[\frac{\xi(n)}{n \log n} \int_{1 / n}^{\delta} \frac{A_{n, \tau}}{t^{2}} d t\right]+o\left[\frac{\xi(n)}{n \log n} \int_{1 / n}^{\delta} \frac{d\left(A_{n, \tau}\right)}{t}\right] \\
& \text { (by hypothesis of the theorem) } \\
& =o(1)+o\left[A_{n, n} \frac{\xi(n)}{\log n}\right]+o\left[\frac{\xi(n)}{n \log n} \int_{1 / \delta}^{n} A_{n, u} d u\right]+o\left[\frac{\xi(n)}{n \log n} \int_{1 / \delta}^{n} u d\left(A_{n, u}\right)\right] \\
& \left(\operatorname{take} \frac{1}{t}=u\right) \\
& =o(1)+o\left[O(1) \frac{\xi(n)}{\log n}\right]+o\left[\frac{\xi(n)}{n \log n}\left(u A_{n, u}\right)_{1 / \delta}^{n}\right] \\
& +o\left[\frac{\xi(n)}{n \log n} \int_{1 / \delta}^{n} u d\left(A_{n, u}\right)\right]+o\left[\frac{\xi(n)}{n \log n} \int_{1 / \delta}^{n} u d\left(A_{n, u}\right)\right] \quad \text { (Integrating by parts) } \\
& =o(1)+o\left[\frac{\xi(n)}{\log n}\right]+o\left[\frac{\xi(n)}{n \log n} n A_{n, n}\right]+o(1)+o\left[\frac{\xi(n)}{n \log n} \int_{1 / \delta}^{n} u d\left(A_{n, u}\right)\right] \\
& =o(1)+o\left[\frac{\xi(n)}{\log n}\right]+o\left[\frac{\xi(n)}{\log n}\right]+o\left[\frac{\xi(n)}{n \log n} n \int_{1 / \delta}^{n} d\left(A_{n, u}\right)\right] \\
& =o(1)+o\left[\frac{\xi(n)}{\log n}\right]+o\left[\frac{\xi(n)}{\log n} \sum_{k=0}^{n} a_{n, k}\right] \\
& =o(1)+o\left[\frac{\xi(n)}{\log n}\right]+o\left[\frac{\xi(n)}{\log n} A_{n, n}\right] \\
& =o(1)+o(1)+o\left[\frac{\xi(n)}{\log n} O(1)\right] \\
& =o(1)+o(1)+o(1), \quad \text { as } n \rightarrow \infty \text {. } \\
& =o(1), \quad \text { as } n \rightarrow \infty \text {. }
\end{aligned}
$$

Lastly, by the Riemann-Lebesgue theorem and the regularity condition of matrix summability, we obtain

$$
I_{4} \leq \int_{\delta}^{\pi}\left|M_{n}(t)\right||d h(t)|=o(1), \quad \text { as } n \rightarrow \infty .
$$


Thus from (6.4), (6.5) and (6.6), we get

$$
t_{n}-\left(-\frac{1}{4 \pi} \int_{0}^{\pi} h(t) \operatorname{cosec}^{2} \frac{t}{2} d t\right)=o(1), \text { as } n \rightarrow \infty .
$$

This completes the proof of the theorem.

\section{Particular Cases.}

(1) If we take $a_{n, k}=\frac{1}{\log (n-k+1)}$ and $\xi(t)=1$ then a result of Tripathi [5] become a particular case of our theorem.

(2) A result of Tripathi and Prasad [6] becomes a particular case of our theorem if $a_{n, k}=\frac{p_{n-k}^{\alpha}}{P_{n}^{\alpha}}$ and $\xi(t)=\frac{t p_{t}^{\alpha} \log t}{P_{t}^{\alpha}}, \alpha \geq-1$. Where $\left\{p_{n}^{\alpha}\right\}$ be a real non-negative non-increasing sequence such that $P_{n}^{\alpha} \rightarrow \infty$, as $n \rightarrow \infty$.

(3) If $a_{n, k}=\frac{p_{n-k}}{P_{n}}$ and $\xi(t)=\frac{\log t \lambda(t)}{P_{t}}$, then a result of Tripathi and Prasad [7] becomes a particular case of main theorem.

(4) If $a_{n, k}=\frac{p_{n-k}^{\varepsilon}}{P_{n}^{\varepsilon}}$ and $\xi(t)=\log t$, where $\left\{p_{n}\right\}$ is a sequence with $p_{0}>0$ and $p_{n} \geq 0$ for $n>0$. For $\varepsilon>-1$, we define $P_{n}^{\varepsilon}=p_{0}^{\varepsilon}+p_{1}^{\varepsilon}+\cdots+p_{n}^{\varepsilon}$, then the main theorem reduces to Tripathi \& Singh [8] theorem.

\section{Acknowledgements}

Authors are thankful to Prof. V. K. Jain, Director, H. B. T. I., Kanpur for encouragement to this work. Shyam Lal one of the author, is grateful to the U. G. C., G. T. Road, Ghaziabad (U. P.), India, for providing financial assistance in the form of minor reasearch project vide letter no f.3.3. (58)/1999-2000/MRP/NR dated 31.03.2000. Authors wish to express their gratitude to the referee for his valuable comments and suggestions for improvement of this paper.

\section{References}

[1] Lal, Shyam, On the degree of approximation of conjugate of a function belonging to wieghted $W\left(L^{p}, \xi(t)\right)$ class by matrix summability means of conjugate series of a Fourier series, Tamkang Jou. of Math, 11(2000), 7-16.

[2] M. L. Mittal and R. Kumar, On matrix summability of Fourier series and its conjugate series., Bull. Cal. Math. Soc., 82(1990), 363-368a.

[3] W. L. Sulaimann, On the Nörlund summability of the conjugate derived series of the Fourier series, The Mathematics Educations, 27(1993), 226-230.

[4] O. Toeplitz, Uberallgemeine Linear Mittelbildunger P. M. F., (1913), 113-119.

[5] L. M. Tripathi, Harmonic summability of conjugate derived series of the Fourier series, Proc. Nat. Acad. Sci. India, 33(1963), 443.

[6] L. M. Tripathi and K. Prasad, On the Nörlund summability of the conjudgate derived series, Indian J. Pure Appl. Math., 4(1972), 58-65. 
[7] L. M. Tripathi and K. Prasad, Nörlund summability of derived Fourier series and conjugate derived Fourier series, Bull. De Laca. Polo. Dex. Sci., 21(1973), 1093-1101.

[8] L. M. Tripathi and A. P. Singh, On the Nörlund summability of the conjugate derived Fourier series, The Jou. of Sci. Res., 31(1980),345-354.

[9] A. Zygmund, Trigonometric series, V. Warszawa (1935), 40-41.

Facility of Sinice, Department of Mathematics, Allahabod University, Allahabod - 211001, Indian. 